\title{
SINTESIS DE NANOPARTICULAS DE OXIDO DE TITANIO EN FASE RUTILO PARA EL DESARROLLO DE UN MATERIAL COMPUESTO DE ESPUMA DE POLIURETANO
}

\section{Synthesis of rutile phase titanium oxide nanoparticles for the development of composite material polyurethane foam}

\author{
Jairo Antonio Cubillos Lobo'; Andrés Albarracín Galindo²; Cesar Ortiz \\ Otálora ${ }^{3}$; Efrén de Jesús Muñoz Prieto ${ }^{4}$
}

\begin{abstract}
${ }^{1}$ Escuela de Ciencias Químicas, Universidad Pedagógica y Tecnológica de Colombia. Grupo Catálisis UPTC.email: jairo.cubillos@uptc.edu.co.

2 Escuela Química, Universidad Pedagógica y Tecnológica de Colombia. Desarrollo y Aplicaciones de Nuevos Materiales (DANUM), e-andres@hotmail.com.

${ }^{3}$ Escuela de Física, Universidad Pedagógica y Tecnológica de Colombia. Grupo de superficies Electroquímica y Corrosión (GSEC), cesaroti@gmail.com.

${ }^{4}$ Escuela de Ciencias Químicas, Universidad Pedagógica y Tecnológica de Colombia. Desarrollo y Aplicaciones de Nuevos Materiales (DANUM), efren17@gmail.com.
\end{abstract}

\section{Resumen}

Este artículo reporta un procedimiento para mejorar las capacidades mecánicas y térmicas de la espuma de poliuretano, buscando desarrollar un material compuesto que pueda ser usado como recubrimiento interior de vehículos y mejore las condiciones de seguridad y confort para sus ocupantes. Para ello, se desarrolló un método de síntesis de nanopartículas de óxido de titanio en fase de rutilo por un proceso sol-gel y posterior tratamiento térmico entre 620 y $700^{\circ} \mathrm{C}$. Se encontró que la fase rutilo es obtenida a $700{ }^{\circ} \mathrm{C}$ y que las nanopartículas presentan un tamaño promedio de $24.7 \mathrm{~nm}$. Estas nanopartículas fueron incorporadas en las espumas de poliuretano. Análisis de microscopía electrónica de rastreo (SEM-EDX), espectroscopia infrarroja con transformada de Fouriel (FT-IR) y ensayos mecánicos de flexión y tracción aplicados sobre la espuma compuesta, mostraron que concentraciones por debajo de $1.5 \%$, cubre puntos huecos en la estructura del poliuretano, generando mejoras en las propiedades mecánicas y térmicas de este material.

Palabras clave: Rutilo, nanopartículas, isocianato, poliol, poliuretano, material compuesto.

\section{Abstract}


170

In this paper, a method for improving the mechanical and thermal capabilities of the polyurethane foam is reported. The proposal is to develop a composite material that can be used as interior lining of vehicles and improve the safety and comfort for its occupants. For this, a synthesis method of nanoparticles of titanium oxide in rutile phase using a sol-gel process and subsequent heat treatment between 620 and 700 ${ }^{\circ} \mathrm{C}$ was developed. It was found that rutilo phase is obtained at $700{ }^{\circ} \mathrm{C}$ and that the nanoparticles have an average size of $24.7 \mathrm{~nm}$. These nanoparticles were incorporated into the polyurethane foam. Analysis of Scanning Electron MicroscopyEnergy Dispersive X-ray Spectroscopy (SEM - EDX), Fourier Transform Infrared Spectroscopy (FT-IR) and mechanical tests of bending and tension applied on the composite foams showed that $\mathrm{TiO}_{2}$ load below $1.5 \% \mathrm{w}$, covering voids in the polyurethane structure, generating improvements in the mechanical and thermal properties of this material.

Keywords: Rutile, nanoparticles, isocyanate, polyol, polyurethane, composite material.

*Para citar este artículo: Cubillos Lobo JA et al. Sintesis de nanoparticulas de òxido de titanio en fase rutilo para el desarrollo de un material compuesto de espuma de poliuretano Revista Bistua. 2016.14(2):169-181

+ Autor para el envió de correspondencia y la solicitud de las separatas: Jairo Antonio Cubillos Lobo. Escuela de Ciencias Químicas, Universidad Pedagógica y Tecnológica de Colombia. GrupoCatálisis UPTC.email: jairo.cubillos@uptc.edu.co. 


\section{Introducción}

Al año mueren alrededor de 1.2 millones de personas en accidentes de tránsito y 50 millones sufren traumatismos que a veces los afectan por el resto de sus vidas. La Organización mundial de la salud (OMS) corrobora que las lesiones generadas por accidentes de vehículos son un problema creciente de salud mundial, por lo que las estadísticas indican que hacia él año 2020, este tipo de accidentes será la tercera causa de mortalidad. Las principales causas de traumatismos en los pasajeros después de ocurrir un accidente de tránsito, están sujetas a los impactos con el interior del vehículo. Además, si el vehículo llega a incendiarse tras el accidente es factible que las piezas en su interior pueden pasar por lo mismo, dependiendo de las propiedades del material con que estén fabricadas. Por tanto, es urgente la imperiosa necesidad de desarrollar nuevos materiales que combinen las propiedades de resistencia, flexibilidad, dureza y que además no mantengan la combustión cuando se les retire la fuente de ignición. Todo lo anterior, ha incentivado la intensión de brindarles seguridad a los ocupantes de los vehículos de transporte, posibilitando la supervivencia en caso de un accidente por impacto.
Existen materiales que pueden reunir los anteriores requisitos, uno de los más importantes es la espuma de poliuretano, material sintético, duroplástico y no fusible, que se obtiene de la mezcla entre isocianato y poliol, dos componentes generados en procesos químicos a partir de petróleo y azúcar (Albán Clavijo, 2012; Papa, 1972). Hay dos maneras de obtener una espuma de poliuretano; la primera de ellas es proyectando al mismo tiempo, sus dos precursores en una superficie y la segunda es por colada, es decir mezclando previamente ambos materiales (Seymour, 1967; Muñoz, 2012). Esta estructura sólida, uniforme y resistente tiene una fórmula celular indicada para su uso como aislante, además posee la capacidad para eliminar los puentes térmicos (Honeywell, 2014; Pérez, 2013). La espuma de poliuretano es un material flexible y maleable para su aplicación como núcleo de paneles con estructuras tipo sándwich, materiales promisorios y de gran interés en la actualidad para el recubrimiento interior de vehículos. También es utilizada como material de impermeabilización (Hilado, 1968).

Por otra parte, se ha encontrado que la adición de nanopartículas de óxido de titanio en fase rutilo que es la fase cristalina que mejor se adapta a la 
estructura del polímero, en concentraciones cercanas al $2 \% p$, mejora las propiedades térmicas y mecánicas de este tipo de materiales.

En la búsqueda de optimizar las propiedades de flexión, tracción y observar el efecto en las propiedades térmicas del material, en este trabajo se sintetizaron nanocristales de $\mathrm{TiO}_{2}$ para ser infundidos en los precursores de espuma de poliuretano y de esta manera obtener materiales compuestos que tengan un uso potencial como recubrimientos para el interior de los vehículos.

\section{MATERIALES Y MÉTODOS}

\subsection{Síntesis de nanopartículas de $\mathrm{TiO}_{2}$}

El óxido de titanio fue preparado por hidrólisis y condensación de isopropóxido de titanio $\left(\mathrm{Ti}\left(\mathrm{OCH}\left(\mathrm{CH}_{3}\right)_{2}\right) 4\right)$ marca Aldrich $97 \%$ de pureza, en alcohol isopropílico $\left(\mathrm{C}_{3} \mathrm{H}_{7} \mathrm{OH}\right)$ marca J.T Baker grado analítico (Murcia, Hidalgo, Navío, Araña, \& Doña-Rodríguez, 2014). Inicialmente se disolvió $192 \mathrm{ml}$ de isopropóxido de titanio en $200 \mathrm{ml}$ de alcohol isopropílico bajo agitación magnética a 700 rpm hasta lograr una mezcla homogénea. Después de esto, se adicionó $200 \mathrm{ml}$ de agua destilada de forma lenta con la ayuda de un cuentagotas. En este procedimiento, la homogenización se logró por utilizar un agitador de vidrio durante las primeras etapas de la
172

disolución. A continuación la suspensión se agitó de manera constante y vigorosa durante 1 hora. Finalizado este tiempo, la mezcla resultante se filtró y el sólido precipitado se recuperó por filtración y se secó a $120{ }^{\circ} \mathrm{C}$ durante 12 horas. Seguidamente, el sólido seco se maceró suavemente usando un mortero de ágata hasta homogenizar el tamaño de partícula. Por último, se tomaron muestras de $2 \mathrm{~g}$ en crisoles diferentes y se sometieron a tratamientos térmicos desde 620 hasta $700^{\circ} \mathrm{C}$ con el fin de observar la influencia de la temperatura de calcinación en la formación de la fase cristalina de rutilo, cuya estructura cristalina se observa en la Figura 1.

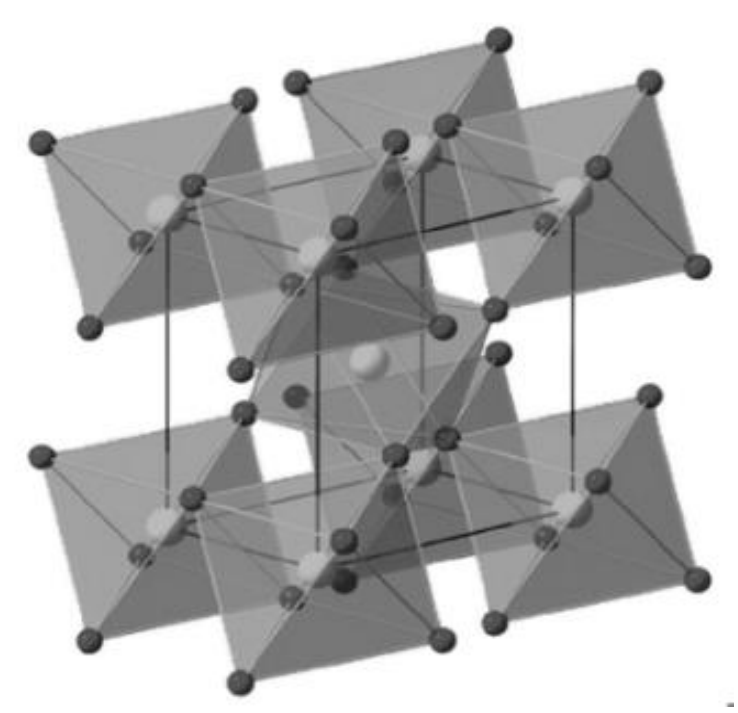

Figura 1. Estructura de la fase cristalina rutilo (Yang et al., 2009) 
El rutilo cristaliza en forma tetragonal distorsionada, presenta gran resistencia al ataque químico y sólo lo atacan los ácidos fluorhídrico y sulfúrico. Se ha utilizado como sustituto del diamante y presenta una menor reactividad que la fase anatasa.

\subsection{Fabricación del núcleo de poliuretano}

Como se aprecia en la Figura 2, la espuma de poliuretano se formó a partir de dos precursores en estado líquido, suministrados por la empresa Espumlatex (Medina, 2014). La parte $A$ es un polímero de isocianato y la parte $B$ es un poliol mezclado con agente de curado y retardante a la llama. Se estudió la adición de nanopartículas de $\mathrm{TiO}_{2}$ de $1,1.5$ y 2 $\%$ p, ya que en estudios previos se encontró que las propiedades de la espuma mejoran con la adición de materiales nanoparticulados que están dentro de este rango de concentraciones (Kabir, Saha, \& Jeelani, 2007; Lake \& Ting, 1999)

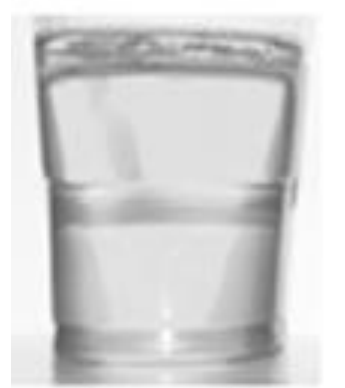

Parte A

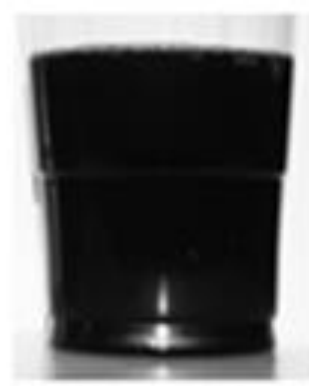

Parte B
173

Figura 2. Precursores para la fabricación de la espuma de poliuretano. Parte A: polímero de isocianato; Parte $\mathrm{B}$ : poliol mezclado con agente de curado y retardante a la llama

Inicialmente, la cantidad de nanopartículas necesarias, se mezcló con el isocianato por ser el componente menos reactivo. Para ello, se utilizó un equipo de cavitación ultrasónico, el cual facilitó la dispersión homogénea de las nanopartículas en el precursor. Para evitar el aumento de la temperatura durante la sonicación, se empleó refrigeración externa, sumergiendo el vaso de precipitados de la mezcla en un baño termostático con una variación de $\pm 1^{\circ} \mathrm{C}$ durante el período de aplicación del ultrasonido. A continuación, el nuevo precursor de isocianato- $\mathrm{TiO}_{2}$ se mezcló con el poliol utilizando agitación mecánica a 2700 rpm durante $20 \mathrm{~s}$. Por último, en un molde de acero inoxidable de 20 $\mathrm{cm}$ de ancho, $30 \mathrm{~cm}$ de largo y $5 \mathrm{~mm}$ de espesor, se vertió la mezcla de precursores y se sometió a una presión por peso de 100 Toneladas por medio de una prensa hidráulica (ver Figura 3). 


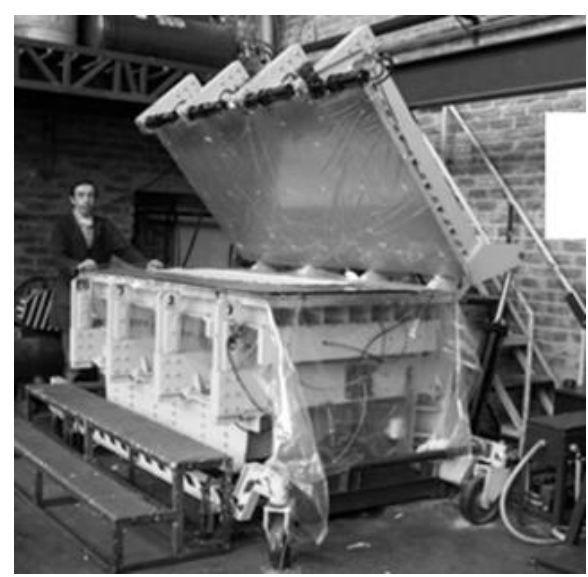

Figura 3. Prensa hidráulica

\section{RESULTADOS}

\subsection{Difracción de rayos $X$ (XRD)}

En la búsqueda de identificar las fases cristalinas presentes en los polvos de óxido de titano después de los tratamientos térmicos, se utilizó DRX, estos análisis se realizaron en un equipo Panalytical XPert PRO MRD.

En los difractogramas (Ver Figura 4) se observa que la presencia del rutilo a $620{ }^{\circ} \mathrm{C}$ está cercana al $95 \%$, mientras que el resto corresponde a la fase anatasa. Al aumentar la temperatura, la presencia de la fase anatasa disminuye hasta desaparecer por completo. El anterior resultado confirma la obtención de óxido de titanio en fase de rutilo a $700^{\circ} \mathrm{C}$.

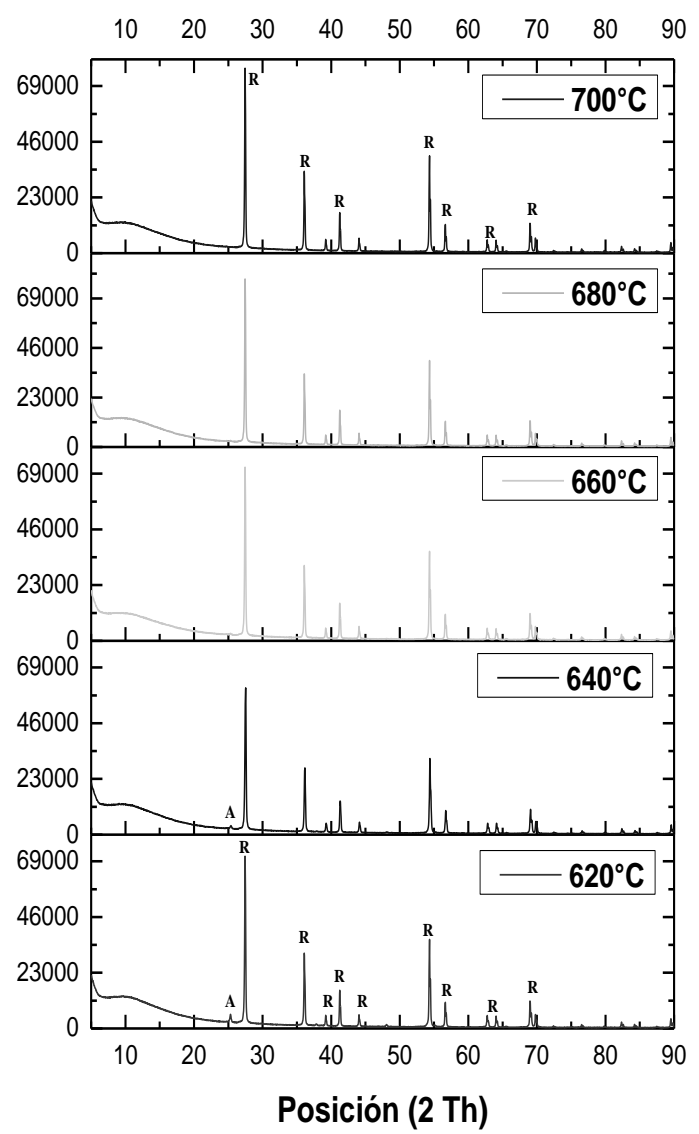

Figura 4. Presencia de $\mathrm{TiO}_{2}$ en fase rutilo al ser sometido a diferentes tratamientos térmicos.

\subsection{Análisis de distribución de tamaño de partícula por dispersión de luz dinámica (DLS)}

Los nanocristales de óxido de titanio sintetizados fueron caracterizados utilizando un analizador de tamaño de partícula por dispersión de luz dinámica (DLS) serie HORIBA LB550. La muestra dispersada en una concentración de $0.1 \%(\mathrm{p} / \mathrm{v})$, fue sometida a sonicación por 2 horas antes de realizar el análisis. La 
temperatura de análisis fue de $25{ }^{\circ} \mathrm{C}$ y el número de lecturas por muestra fue de 5 . Los resultados se resumen en la Tabla 1 y Figura 5. Estos resultados muestran que el tamaño promedio de los nanocristales sintetizados es de $24.7 \mathrm{~nm}$, con lo cual se demostró la estandarización del método de síntesis y los tratamientos térmicos para la obtención de $\mathrm{TiO}_{2}$ en fase rutilo.

Tabla 1. Resultados obtenidos al realizar el análisis de distribución de tamaño de la muestra de $\mathrm{TiO}_{2}$

\begin{tabular}{|c|c|c|c|c|}
\hline Muestra & Media & Mediana & Desviación & $\begin{array}{c}\text { Coeficiente } \\
\text { Estándar }\end{array}$ \\
& $(\mathbf{n m})$ & $(\mathbf{n m})$ & $\begin{array}{c}\text { de } \\
(\mathbf{n m})\end{array}$ & $\begin{array}{c}\text { variación } \\
(\%)\end{array}$ \\
\hline $\mathrm{TiO}_{2}$ & 24.7 & 23.1 & 12.04 & 48.68 \\
\hline
\end{tabular}

Figura 5. Distribución de tamaño para la muestra de $\mathrm{TiO}_{2}$

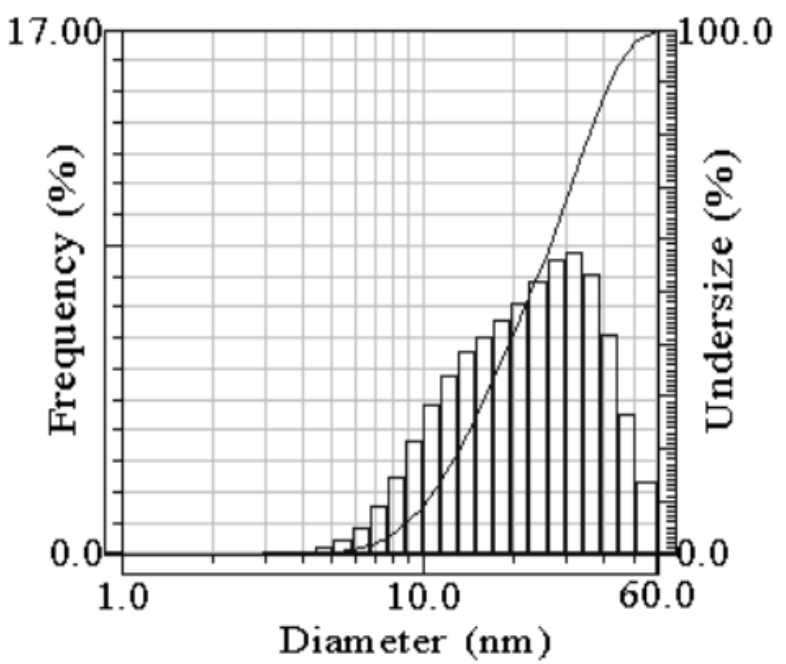

175

\subsection{Análisis termogravimétrico (TGA)}

Para medir la estabilidad térmica de la espuma de poliuretano infundida con las nanopartículas de $\mathrm{TiO}_{2}$, se llevaron a acabo análisis termogravimétricos (ver figura 6). Estas pruebas se realizaron en un equipo Marca T.A Instruments, modelo TGA Q500. El análisis se realizó en atmósfera de nitrógeno tipo 4.5, con una rampa de calentamiento de $10^{\circ} \mathrm{C} / \mathrm{min}$ desde temperatura ambiente $\left(22^{\circ} \mathrm{C}\right)$ hasta $700^{\circ} \mathrm{C}$. El material fue cortado en pequeños trozos cuadrados de $1 \mathrm{~mm}$ de lado y luego cantidades entre 9 y $23 \mathrm{mg}$ de material, se colocaron en un soporte redondo de platino para su posterior análisis. Se observó la degradación del material durante la prueba y se tomaron las temperaturas correspondientes para cada porcentaje de descomposición.

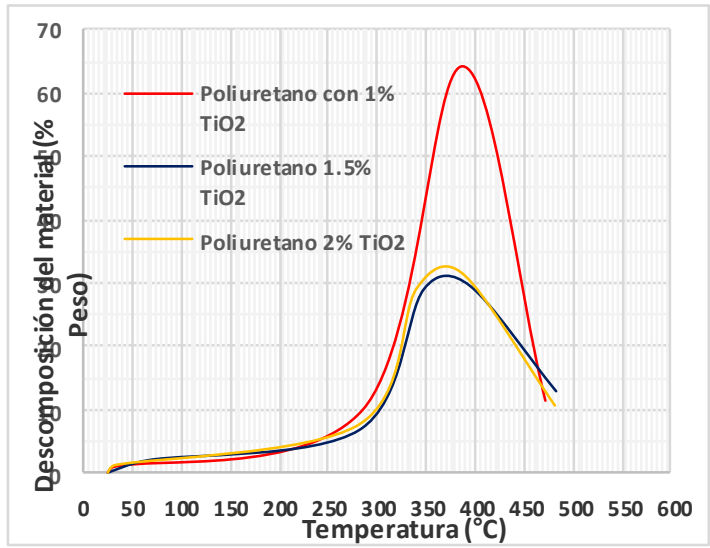


Figura 6. Análisis termogravimétrico de los materiales obtenidos.

Como puede observarse en las Figura 6 a medida que se aumenta la concentración de nanopartículas en el núcleo de poliuretano, el material se vuelve más resistente a aumentos de temperatura, es decir el material se descompone lentamente, con esto se comprueba que la adición de $\mathrm{TiO}_{2}$ provee a la espuma una mejora en sus propiedades térmicas. Cabe resaltar que al comparar el poliuretano obtenido con $1.5 \%$ p de $\mathrm{TiO}_{2}$ con aquel que contiene $2 \% p$, no hay cambios significativos en las propiedades térmicas, sugiriendo que la mejora sustancial se da a concentraciones por debajo de 1.5 $\%$ p de $\mathrm{TiO}_{2}$.

\subsection{Microscopía Electrónica de Barrido (SEM-EDX)}

Figura 7. Imágenes mapa composicional del poliuretano infundido con $1 \%$ de $\mathrm{TiO}_{2}$ en fase rutilo: (a) Vista superior, (b) Distribución de Ti, (c) Distribución de C, (d) Distribución de 0 (a)

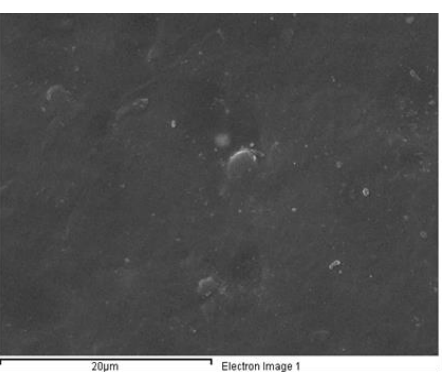

(c)

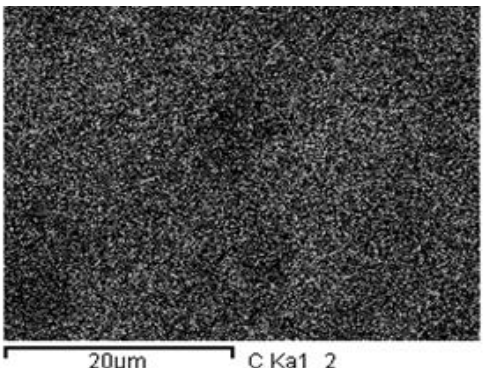

$20 \mu \mathrm{m}$ CKa1 2 (b)

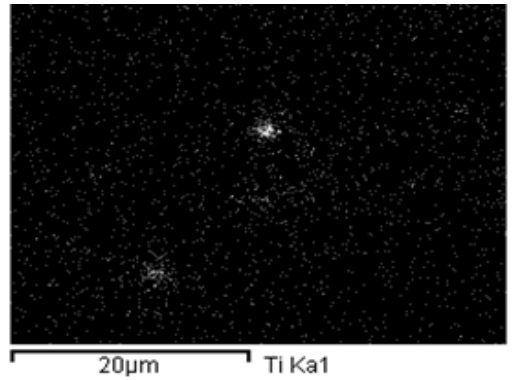

(d)

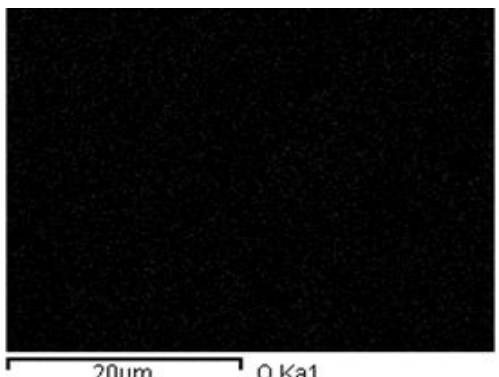

$20 \mu \mathrm{m}$ OKa1

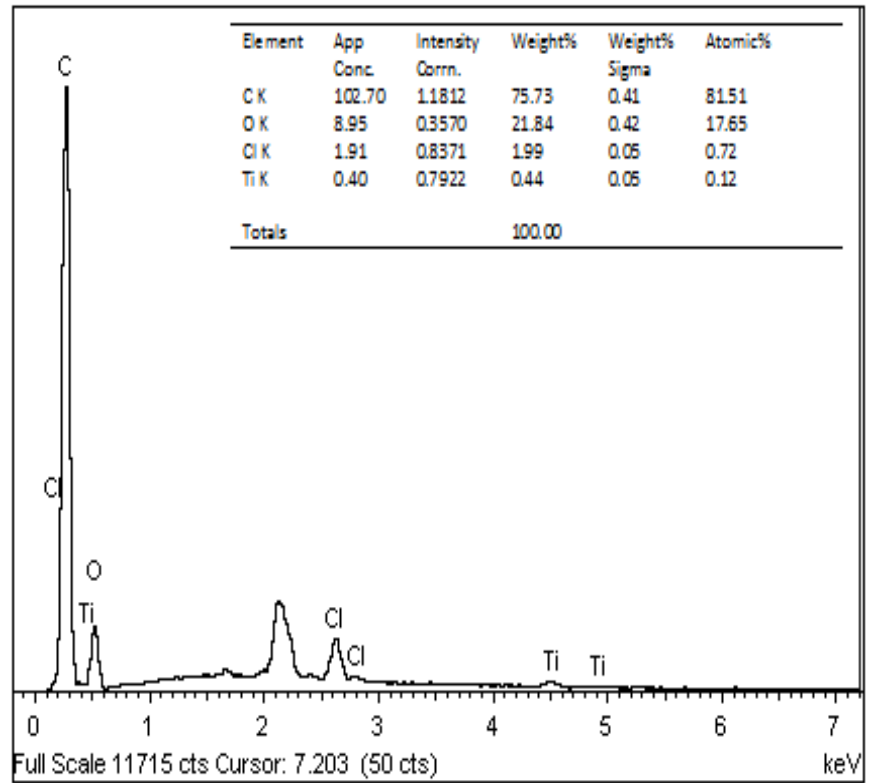

Figura 8. Espectro composicional poliuretano infundido con el $1 \% \mathrm{p} \mathrm{TiO} 2$ 
Las imágenes de la Figura 7, muestran que la adición de nanocristales en concentraciones al 1 $\% p$ al precursor de la espuma origina una dispersión de estos de forma homogénea en el material compuesto, además no se observan deformidades en la superficie.

(a)
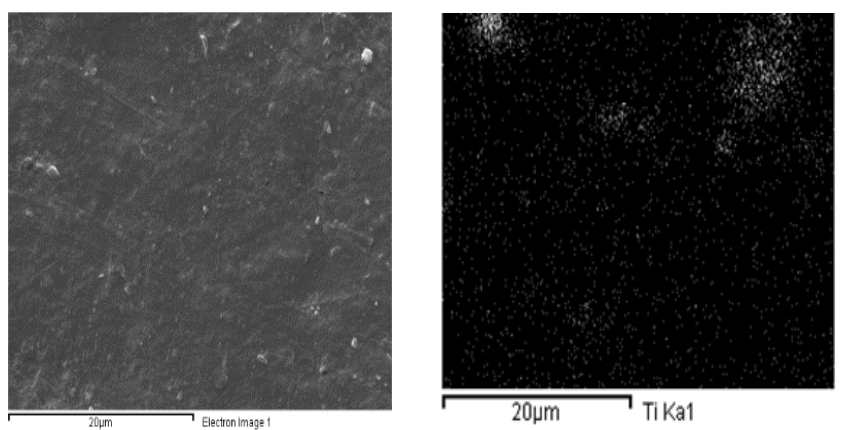

(c)

(d)

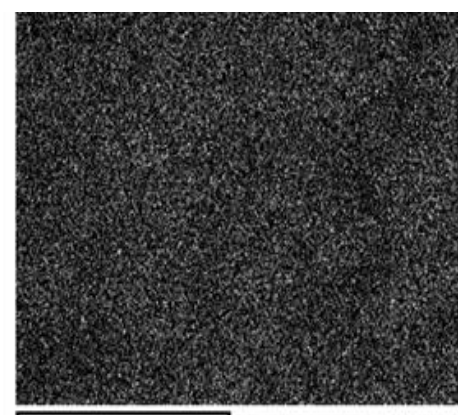

$20 \mu \mathrm{m}$ CKa1_2
177

Figura 9. Imágenes mapa composicional del poliuretano infundido con $2 \%$ de $\mathrm{TiO}_{2}$ en fase rutilo: (a) Vista superior, (b) Distribución de Ti, (c) Distribución de C, (d) Distribución de O.

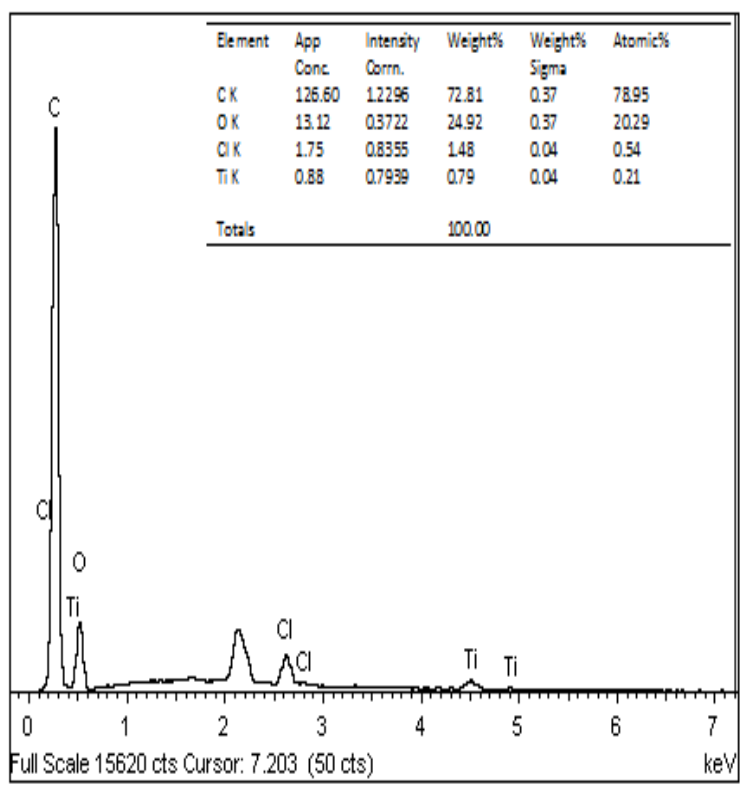

Figura 10. Espectro composicional poliuretano infundido con el $2 \% \mathrm{p} \mathrm{TiO}_{2}$.

Mientras que las imágenes arrojadas por el mapeo composicional (ver Figura 9) y que corresponden a adiciones de $\mathrm{TiO}_{2} 2 \%$ peso, muestran una mayor acumulación de las nanopartículas en ciertos sectores del material, provocada por el exceso de $\mathrm{TiO}_{2}$. Lo anterior confirma que concentraciones máximas a $1.5 \% \mathrm{p}$ de $\mathrm{TiO}_{2}$, el nanocompuesto cubre los espacios libres en la celda del polímero y por encima de dicha concentración se genera un exceso del compuesto que crea defectos en 
la superficie del material, como puede observarse en la imagen 9(a) donde se nota la rugosidad que esto genera.

\subsection{Espectroscopia Infrarroja con Transformada de Fourier (FT-IR)}

FT-IR se utilizó para determinar la unión química entre las nanopartículas y las cadenas del polímero. Se analizaron las estructuras de dominio de ambos arreglos, es decir las espumas con y sin adición de nanopartículas. Para ello, se usó un espectrofotómetro infrarrojo con transformada de Fourier Prestige-21 marca Shimadzu.

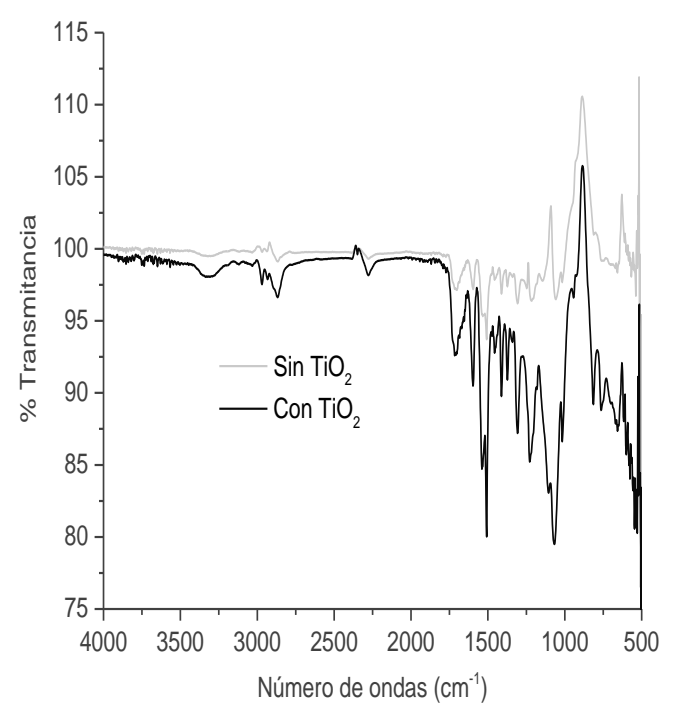

Figura 11. Espectro infrarrojo para el núcleo de poliuretano con y $\sin \mathrm{TiO}_{2}$ en fase rutilo
178

Como se puede observar en la Figura 11 , coinciden la posición de los picos distintivos para la espuma de poliuretano infundida y la espuma de poliuretano. Lo anterior indica que la estructura de la espuma de poliuretano no ha sido afectada por la presencia de las nanopartículas de $\mathrm{TiO}_{2}$ en fase rutilo. Lo anterior sugiere que la titania no reacciona con las moléculas presentes en la espuma en porcentajes de hasta $2 \%$, que fueron precisamente los empleados en este trabajo.

\subsection{Ensayos de flexión y tracción}

Para determinar que mejoras en las propiedades mecánicas se dan en la espuma tras la infusión de las nanopartículas, se realizaron pruebas de flexión y tracción en una maquina universal de ensayos. Las dimensiones de la probeta para los ensayos de tracción fueron: espesor $=4 \mathrm{~mm}$, ancho $=12,7 \mathrm{~mm}$, longitud = $64 \mathrm{~mm}$ y para los ensayos de flexión: espesor $=4,8 \mathrm{~mm}$, ancho $=25 \mathrm{~mm}$ y de longitud $125 \mathrm{~mm}$ (Ver Tabla 2).

Tabla 2. Tipo de muestra usada para el ensayo. 


\begin{tabular}{|c|c|}
\hline Muestra 1 & $\begin{array}{c}\text { Lamina de poliuretano } \\
\text { (PUR) }\end{array}$ \\
\hline Muestra 2 & PUR $-1 \% \mathrm{TiO}_{2}$ \\
\hline Muestra 3 & PUR $-1,5 \% \mathrm{TiO}_{2}$ \\
\hline Muestra 4 & PUR $-2 \% \mathrm{TiO}_{2}$ \\
\hline
\end{tabular}

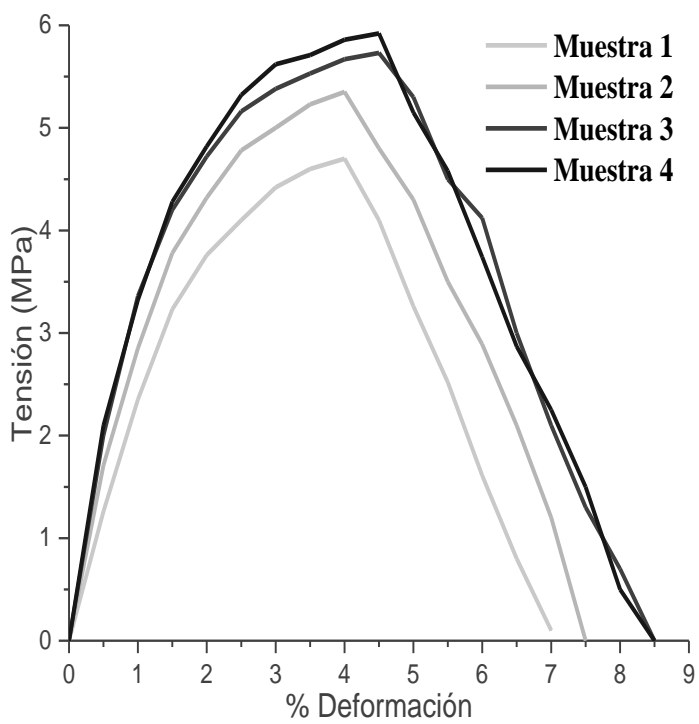

Figura 12. Curva tensión vs deformación ensayos de tracción

Según los ensayos (ver Figura 12) se observa que al someter las probetas muestra de material compuesto al esfuerzo axial de tracción creciente, ocurre más rápidamente la rotura de la probeta de poliuretano si se compara con alguna muestra $(2,3$ y 4) de poliuretano infundido con $\mathrm{TiO}_{2}$, esto se debe a que las nanopartículas de óxido de titanio de $24.7 \mathrm{~nm}$ de tamaño promedio (Ver Tabla 1)
179

cubren los huecos en la estructura del polímero generando una celda más compacta, lo que mejora su resistencia a la fuerza estática aplicada lentamente. Por otra parte, la Figura 15 indica que al aumentar la concentración de $\mathrm{TiO}_{2}$ Rutilo de 1 al $1.5 \%$ mejora significativamente las propiedades de resistencia mecánica, mientras que al aumentar la concentración de 1.5 a $2 \% p$ de titania no referencia cambio significativo. Lo anterior sugiere que los espacios estructurales en la espuma, son llenados por las nanopartículas y por encima de estas concentraciones, estas pasan a convertirse en un material remanente dentro de la estructura.

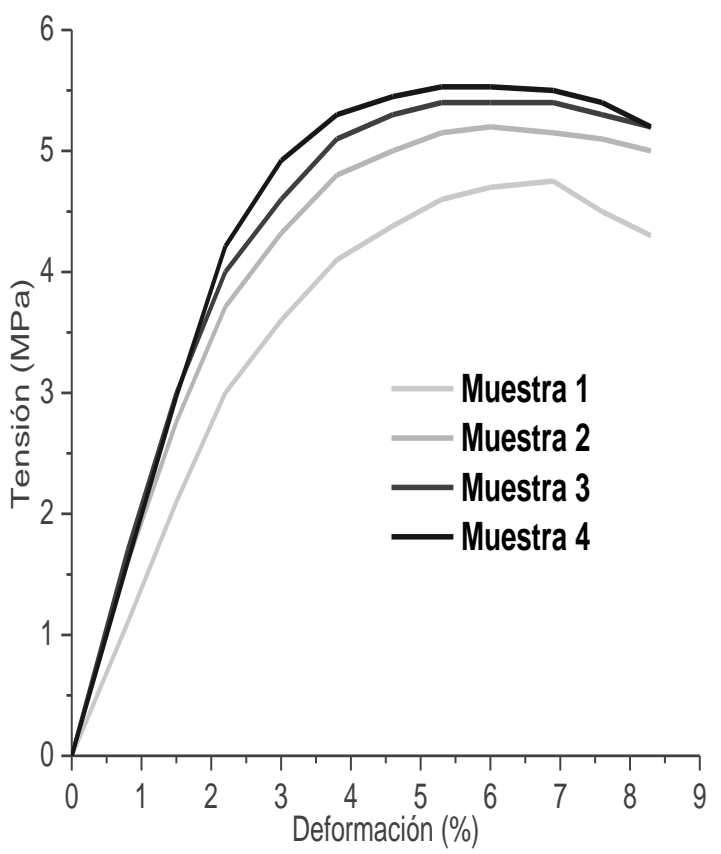


Figura 13. Ensayos de flexión: curva tensión vs deformación.

En los ensayos realizados a las probetas del material compuesto, se desea evaluar la influencia de las nanopartículas en el estiramiento de la probeta entre dos puntos fijos de la misma, a medida que se incrementaba la carga aplicada. En la Figura 13, podemos observar que la deformación elástica de la muestra de poliuretano ocurre más rápidamente que la de poliuretano infundido, lo mismo ocurre en los puntos de fluencia o cedencia donde se observa que la deformación brusca de la probeta sin incremento de la carga aplicada, ocurre más rápido en el poliuretano. Lo anterior, reconfirma que los espacios en la celda del polímero son llenados por los nanocristales, por lo que obtenemos un material más compacto que aumenta la resistencia a esfuerzos mecánicos.

\section{CONCLUSIONES}

Es muy importante desarrollar materiales que ofrezcan avances significativos en la búsqueda de seguridad o disminución de los riesgos de muerte o traumatismos de los pasajeros en caso de accidente de vehículo.
180

Los materiales desarrollados como estructuras tipo sándwich, como el perfeccionado en este trabajo de investigación elevan de manera significativa las probabilidades de sobrevivir a un accidente de vehículo, ya que presentan buena resistencia al impacto, altas temperaturas y elevan su costo-beneficio en cuanto a su larga vida útil.

El método de síntesis de óxido de titanio en fase rutilo utilizado en este trabajo, permite obtener nanopartículas con tamaños de partícula promisorios en la búsqueda de fabricar materiales de elevada resistencia mecánica y térmica.

Con la dispersión de óxido de titanio en fase rutilo, se logra un aumento en la propiedades mecánicas y térmicas de la espuma de poliuretano y se demuestra que estas cubren los espacios que se generan dentro de la estructura del material.

\section{AGRADECIMIENTOS}

Los autores agradecen a la Dirección de Investigaciones de la Universidad Pedagógica y Tecnológica de Colombia (DIN-UPTC) por el apoyo financiero a través del proyecto SGI 1776.

\section{Referencias bibliogràficas}


Albán Clavijo, B. G. M., Edwin. (2012). Estudio de paneles de fibropoliuretano para uso en mamposterias. (Pregrado), Universidad Politécnica Salesiana, Ingeniería Civil.

Hilado, C. J. (1968). Analysis of Some Flammability Characteristics of Cellular Polymers. I\&EC Product Research and Development, 7(2), 8193.

Honeywell. (2014). El aislamiento y el PUR. Características y aplicaciones. Retrieved 27/10/2014, 2014, from http://www.saizmartinez.es/pdf/caract eristicas-aplicaciones.pdf

Kabir, M. E., Saha, M. C., \& Jeelani, S. (2007). Effect of ultrasound sonication in carbon nanofibers/polyurethane foam composite. Materials Science and Engineering: A, 459(1-2), 111-116.

Lake, M. L., \& Ting, J.-M. (1999). Chapter 5 - Vapor Grown Carbon Fiber Composites. In T. D. Burchell (Ed.), Carbon Materials for Advanced Technologies (pp. 139-167). Oxford: Elsevier Science Ltd.

Medina, D. (2014) Flamabilidad de los materiales interiores de vehiculos automotores. (pp. 1-2).
Muñoz, E., Palacios, Joaquín \& Alonso, Carlos. (2012). Microwave and Ultrasound Activation Effect on Cationization of Corn and Potato Starches. Rev. Ciencia en Desarrollo, 4(1), 151-173.

Murcia, J. J., Hidalgo, M. C., Navío, J. A., Araña, J., \& Doña-Rodríguez, J. M. (2014). Correlation study between photo-degradation and surface adsorption properties of phenol and methyl orange on $\mathrm{TiO} 2$ Vs platinumsupported TiO2. Applied Catalysis B: Environmental, 150-151(0), 107-115.

Papa, A. J. (1972). Flame Retardation of Polyurethane Foams in Practice. Product R\&D, 11(4), 379-389.

Pérez, M. E., Ruiz D.M., Schneider, M., Autino, J. C \& Romanelli, G. (2013). La química verde como fuente de nuevos compuestos para el control de plagas agrícolas. Rev. Ciencia en Desarrollo, 4 (2), 83-91.

Seymour, R. (1967). PLASTICS TECHNOLOGY. Industrial \& Engineering Chemistry, 59 (8), 62-74.

Yang, Z., Choi, D., Kerisit, S., Rosso, K. M., Wang, D., Zhang, J., . . . Liu, J. (2009). Nanostructures and lithium electrochemical reactivity of lithium titanites and titanium oxides: A review. Journal of Power Sources, 192(2), 588-598. 
